\title{
A Multi-Reconfigurable CLL-Loaded Planar Monopole Antenna
}

\author{
Mohammad M. FAKHARIAN ${ }^{1}$, Pejman REZAEI ${ }^{2}$, Ali A. OROUJI ${ }^{2}$ \\ ${ }^{1}$ Faculty of Engineering, University of Garmsar, Garmsar, 35817-55796, Iran \\ ${ }^{2}$ Dept. of Electrical and Computer Engineering, Semnan University, Semnan, 35131-19111, Iran \\ mmfakharian@ugsr.ir,prezaei@semnan.ac.ir, aliaorouji@semnan.ac.ir
}

Submitted October 9, 2019 / Accepted March 10, 2020

\begin{abstract}
In this paper, multi-reconfiguration capabilities of a planar monopole antenna with two switchable capacitively loaded loops (CLLs), as near field resonant parasitic elements, are introduced. The idea is to apply the CLLs not only to minimize the dimensions of the antenna, but also to present multiple resonances, which can be satisfactorily chosen by applying switches placed across six gaps of the CLLs. By changing the switched states, it is feasible to obtain different reconfigurations such as frequency agility (from 1.5 to $2.9 \mathrm{GHz}$ ), polarization diversity (with circular polarization bandwidth from 1.59 to $1.72 \mathrm{GHz}$ ), and various shapes of the radiation patterns and beam directions (change in the $\pm 30^{\circ} y$-direction) of the antenna. The transmutation of polarization designs from their linear counterparts to left hand and right hand circular polarizations by introducing an asymmetry in the configuration of the twoCLLs is also represented. The prototypes of the proposed antenna are fabricated and tested. The measured reflection coefficient, radiation pattern, gain and axial ratio results are presented and compared to the corresponding simulated values.
\end{abstract}

\section{Keywords}

Multi-reconfigurable antenna, resonant parasitic element, monopole

\section{Introduction}

Reconfigurable systems are the desirable ideas for the future radio networks, which are the basic principle in software-defined radios, cognitive radios, cooperative networks, and etc. [1]. There is a need for multifunctional antennas for these challenges. The reconfiguration is achieved by techniques that redistribute the flow of current on the surface of the antenna and by means of that adapting to alter the electromagnetic fields and performance characteristics of the antenna's effective aperture. Typically, the reconfiguration techniques are obtained by merging of ideal switches [2] or electronic switching components such as: PIN diodes [3-8], varactor diodes [9] and RF-MEMS [10] on the configuration of the antenna.
The property of the reconfigurable antennas is to react automatically by altering the resonant frequency, polarization and radiation pattern. Frequency reconfigurable antennas allow efficiently sense the frequency, dynamic spectrum allocation, and potential to reduce the size of the front end system. However, radiation pattern reconfigurable antennas can be applied to avoid noisy environments, inband interference, and increase the capacity and security of the system. Polarization reconfigurable antennas are also applied to filter the detrimental fading loss caused by the multipath effects. Thus, these reconfigurable antennas can be diversified in frequency [2, 5, 8], radiation pattern [3], [4], polarization [6] or combinations of these characteristics [10-14] that are called multi-functional/hybrid/multireconfigurable antennas.

Recently, there is a strong push for multi-reconfigurable antennas with the independent or simultaneous reconfiguration of the operating frequency, radiation pattern and polarization [10], [12-14]. One of the approaches to achieve this type of multi-reconfigurable is the use of pixel antennas [10]. For example, an $n_{\mathrm{px}} \times n_{\mathrm{px}}$ matrix of metallic pixels antenna interconnected through MEMS switches presented to operate within the 4-7 GHz frequency band [10]. The second approach in this type of multi-reconfigurable is the use of parasitic pixel layer [12], where the reconfiguration mechanism is based on the same pixel antenna concept. In [12], a diode-switched grid of small metallic patches is used as a parasitic layer on top of the antennas acting as driven patch to provide reconfiguration capabilities. However, these designs are much complex in structure and fabrication trouble with the usage of more switching elements and vias, which seriously affects on the antenna efficiency and cost. Recently, there is a demonstration of a single radiating element at two distinctive frequencies with different polarization and radiation pattern characteristics for WLAN application [13]. The antenna made of four radiating patches of dimensions $5 \times 4 \mathrm{~mm}^{2}$ that are interconnected through three switches to get different modes with the desired reconfigurability. In this study, coplanar waveguide (CPW) feeding technique is used to enhance the impedance bandwidth of the antenna. However, this work leads to a low reconfigurability and thereby giving few operating modes with relatively similar 
characteristics, especially in the radiation patterns. Moreover, the reconfiguration mechanism in [13] is based on the same pixel antenna concept with larger pixels with simultaneous reconfiguration of the pattern and polarization. Newly, a substrate integrated waveguide (SIW) cavitybacked slot antenna, with polarization, frequency, and pattern diversities, is designed [14]. The reconfigurability of this design is obtained by controlling the states of 12 pairs of switches between two crossed slots etched on both top and bottom metal surfaces of the SIW cavity. However, this design applies a lot of switches and parasitic elements in the complicated structure that affects on the RF radiation of the antenna. The design is also shown a unidirectional radiation performance for the various different states, while an omnidirectional radiation is required for a lot of applications.

Our approach to achieve a multi-reconfigurable function is the use of metamaterial-inspired antennas [15], [16]. So far, different types of the metamaterial-inspired antennas, which are electrically small antennas, efficient and inherently matched to the source, have been presented to have multifunctional properties. For example, a circular polarization and multiband antenna using near field resonant parasitic (NFRP) elements has been designed [17]. A variety of these designs have comprised the introduction of split ring resonator (SRR) and capacitively-loaded loop (CLL) elements as the NFRP elements [15-20]. In the work [18], the structures are reported in which a driven monopole antenna integrated with one and two switchable CLL elements that comprise two open-closed configurations, to achieve just the reconfigurable patterns. In the [19], the frequency and pattern diversities of a printed monopole antenna loaded with a couple of SRRs at the two sides of it is presented. The operation of the antenna is achieved by four ideal switches placed across the gaps of the SRRs. In the work [20], a frequency-agile circularly polarized (CP) NFRP antenna design is obtained by inserting two varactors into its two orthogonal, CLL-based NFRP elements.

Inspired by previous works [18-20], in this paper, a monopole planar antenna loaded with two reconfigurable CLL-based NFRP elements printed on the same board and placed at the two sides of it is proposed. The mechanism of coupling antenna is primarily magnetically-driven and it has been depicted as a magnetic excitation of the magnetic resonance (MEMR) [17]. In addition to miniaturization, the main idea is to embed six switches across the CLLs, to make independent multi-reconfigurable capabilities, including frequency agility, pattern reconfigurability, and polarization diversity. A functional prototype is proposed that accretion and compact characteristics make it appropriate for wireless applications. In contrast to existing designs $[10,12,14]$, the antenna requires a reduced number of switches and low complexity. The antenna has also very small dimensions. The size in each dimension is less than $0.25 \lambda_{0}$ of the lower frequency $\left(\lambda_{0}\right.$ is the free-space wavelength at $1.5 \mathrm{GHz}$ ). In comparison to the previous report [13], the CLL elements as the NFRP elements achieve much

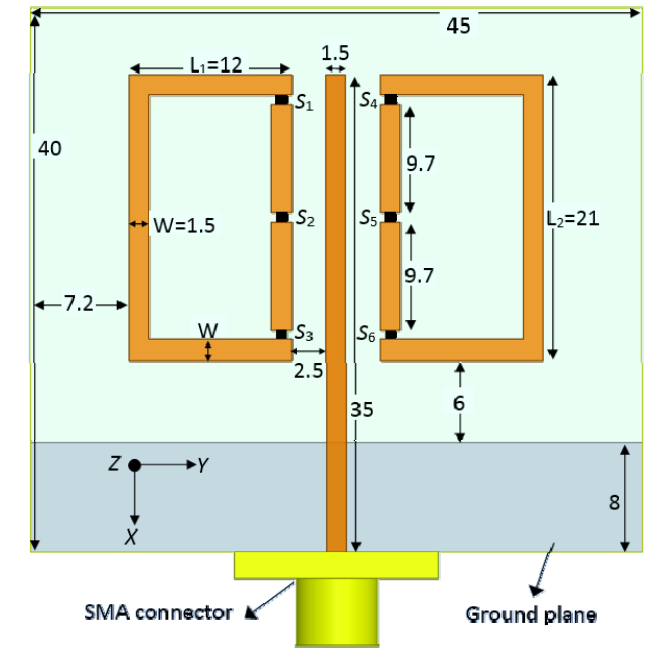

Fig. 1. Geometrical structure of the proposed multireconfigurable antenna. (Unit: $\mathrm{mm}$ ).

higher radiation efficiencies rather than rectangular patches. Furthermore, the antennas reported here could be integrated into various vehicles and other transportation systems.

\section{Antenna Design}

Figure 1 shows the basic structure of the proposed antenna with switched two-CLL NFRP elements. It is etched on an FR4 substrate with relative permittivity 4.4, thickness $1.6 \mathrm{~mm}$, and size of $45 \times 40 \mathrm{~mm}^{2}$. In order to increase the inductive load, the distance between the driven monopole antenna and the NFRF elements is decreased, and thus the overall dimensions of the antenna are minimized. To obtain the multi-reconfigurable, the structure of the two-CLL NFRP elements are modified to (1) alter the effective length of the NFRP elements for achieving operating band/frequency agility and (2) alter the current flow direction for changing the spatial distribution of radiation which in turn affects at the polarization of the structure. For this purpose, six switches namely $\mathrm{S}_{1}, \mathrm{~S}_{2}, \mathrm{~S}_{3}, \mathrm{~S}_{4}, \mathrm{~S}_{5}$ and $\mathrm{S}_{6}$ are applied in the two CLL elements in various configurations. The ON and OFF states of each switch correspond to an open-CLL (open circuit for the CLL-gap) and a closed-CLL (short circuit for the CLL-gap), respectively.

In this study, the six switches are realized as metal bridges. The absence of the metal bridge is treated as OFF and the presence as ON [2]. The dimension of the ideal switch is selected $0.7 \times 0.2 \mathrm{~mm}^{2}$ to realize the actual dimension of an RF PIN diode switch. Signal transmission is done by an SMA connector attached to the monopole antenna. The driven monopole is excited with a $50-\Omega$-microstrip feed line. The optimized dimensions of the antenna are given in the same Fig. 1.

The proposed structure has been simulated using the numerical full-wave ANSYS-HFSS software, and the results of the simulations showing the different operating modes are provided and summarized in the next section. 


\begin{tabular}{|c|c|c|c|c|c|c|c|}
\hline \multirow{2}{*}{$\begin{array}{c}\text { Mode/ } \\
\text { Antenna }\end{array}$} & \multicolumn{6}{|c|}{ Switch status } & \multirow{2}{*}{$\begin{array}{l}\text { Reconfigura- } \\
\text { bility }\end{array}$} \\
\hline & $\mathrm{S}_{1}$ & $\overline{S_{2}}$ & $\overline{\mathrm{S}_{3}}$ & $\mathrm{~S}_{4}$ & $\overline{S_{5}}$ & $\mathrm{~S}_{6}$ & \\
\hline Ant. 1 & 1 & 1 & 1 & 1 & 1 & 1 & \multirow{4}{*}{ Frequency } \\
\hline Ant. 2 & 1 & 1 & 0 & 1 & 1 & 0 & \\
\hline Ant. 3 & 1 & 0 & 0 & 1 & 0 & 0 & \\
\hline Ant. 4 & 0 & 1 & 0 & 0 & 1 & 0 & \\
\hline Ant. A & 1 & 0 & 1 & 1 & 1 & 1 & \multirow{2}{*}{$\begin{array}{c}\text { Radiation } \\
\text { Pattern }\end{array}$} \\
\hline Ant. A' & 1 & 1 & 1 & 1 & 0 & 1 & \\
\hline Ant. $\alpha$ & 1 & 1 & 0 & 0 & 1 & 1 & \multirow{2}{*}{ Polarization } \\
\hline Ant. $\alpha^{\prime}$ & 0 & 1 & 1 & 1 & 1 & 0 & \\
\hline
\end{tabular}

Tab. 1. Antenna possible configurations for the different combinations of the switch status (0: OFF and 1: ON).

\section{Multi-Reconfiguration Performance}

The proposed antenna can be operated as a multimode antenna to have different polarizations and radiation patterns while keeping the same operating frequency/band. As a multiband frequency tunable antenna, the proposed antenna can be used to adapt its operating frequency (while keeping the same radiation pattern and polarization) into different bands to serve distinct wireless applications. With this functionality, the antenna can be also used to excite distinct radiation patterns with a specific polarization while maintaining the operating frequency unchanged.

Due to the ON/OFF status of the six switches, thirtysix configurations have been investigated and simulated. Only eight of them lead to interesting results (i.e., it alters the operation frequency of the antenna while keeps an acceptable matching level) and the corresponding reconfigurabilities of them in the frequency, polarization or radiating pattern have been shown in Tab. 1 .

\subsection{Frequency Agility}

We expect that the proposed antenna works in two operation modes: one due to the monopole and one due to the excitation of two-CLL NFRP elements. The resonant frequency of the CLL elements is mainly independent of the dimensions of the monopole and is mostly related to the geometrical dimensions of CLLs [16], [17]. Therefore, the CLLs are subwavelength resonators, so that with proper designing their dimensions, they can be achieved at much lower resonant frequency than the unloaded planar monopole.

In order to understand the coupling mechanisms and performance of the antenna, circuit model of the CLLbased NFRP antenna is explained and developed. The twoCLL NFRP elements are excited by the magnetic fields, i.e., the CLL elements are driven by the magnetic field component produced using the driven monopole. It has a vast net magnetic flux through the CLLs while the effects of electric driven gap are insignificant [18]. The magnetically-coupled model based on the results of the magneticbased metamaterial incorporation is shown in Fig. 2. Through the inductor, the source is the flux of the magnetic

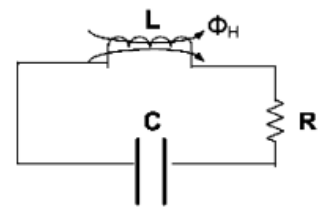

Fig. 2. Magnetically-coupled circuit model for the CLL-based NFRP antenna [18].

field, $\phi_{\mathrm{H}}$. Therefore, the CLL elements act like a series RLC circuit that driven using a source of current.

The overall size of the CLL elements, their trace widths $(W)$, their capacitive gaps (open or short-circuited) and the height of their legs $\left(L_{1}\right.$ and $\left.L_{2}\right)$ control their resonant frequencies. These design variables, along with the monopole dimensions, have achieved an enough degree of freedom to change the antenna operating to obtain multiple frequencies and high radiation efficiencies with either linear (LP) or circular polarization (CP) operation. The thicker $W$ produces a smaller effective inductance $L_{\text {eff. }}$ The larger $L_{1}$ and $L_{2}$ also lead to a smaller flux area of the radiating semi-loop, and for this reason made a smaller effective inductance $L_{\text {eff. }}$ Therefore, when the size of the leg is enlarged, at the first the resonant frequency takes lower and then takes higher as the size of the leg is increased to a level where it negatively effects on the value of inductance [18]. On the other hand, we can control the effective capacitance $C_{\text {eff }}$ by making the open or short-circuited across the legs. In particular, since the capacitors produced by the open-circuited are then in series, their overall capacitance is lower than the value of the original. Since the resonance frequency is

$$
f_{\text {res }}=\frac{1}{2 \pi} \frac{1}{\sqrt{L_{\text {eff }} C_{\text {eff }}}}
$$

and the total effective capacitance has been reduced, the frequency of resonance increases. In order to obtain this, the switches are inserted in the CLL radiators, as shown in Fig. 1. This allowed us to modify the capacitance of gap without having to redesign the CLL configuration to work the antenna in various different operating modes. In this regard, it is shown that by augmenting the NFRP structures of the antenna with a pair of varactors, the corresponding frequency-agile version is obtained [20]. Moreover, a thinner loop width yields a higher resistance, which in turn means that it makes a larger conductive loss $R_{\mathrm{L}}$. On the other hand, a thinner loop width also produces a larger effective radiation loop area, which in turn yields a mutually larger radiation resistance $R_{\mathrm{r}}$. The radiation efficiency $R_{\mathrm{E}}=R_{\mathrm{r}} /\left(R_{\mathrm{r}}+R_{\mathrm{L}}\right)$ and, hence, the $Q_{\text {ratio }}$ depends on these terms.

\subsection{Radiation Pattern Reconfigurability}

It is found from the far-field radiation pattern and the current distribution that the one gap CLL-based NFRP antenna had an asymmetric radiation pattern and radiated in a mixed magnetic and electrical resonance mode [18]. 
The asymmetric radiation is clarified using the asymmetric distribution of the current on the CLL, i.e., it increases (decreases) as it access the gap in the CLL. The overall current producing the radiated field can be divided into the current on the monopole element and the "loop current" on the CLL element. The loop current on the CLL element controls the behavior. Therefore, to obtain the symmetric (asymmetric) radiation pattern, it has noticed that the symmetric (asymmetric) CLL element would be required.

When the switch status is such that one of the two CLL elements has three short-circuit gaps (inactive), while the other one is active, the frequency of resonance related to the value of the single-CLL-loaded monopole antenna, as well as its radiation. Thus, depending on the active CLL, the radiation pattern pointing either along the negative or positive of the $y$-axis can be obtained. According to Tab. 1, due to the symmetry of the antenna structure, the resonance frequency for Ant. A is the same result of Ant. A', while, the direction of their beam is contrary.

\subsection{Polarization Diversity}

By introducing quasi-symmetry or asymmetry in the configuration of the two-CLL NFRP elements, one can obtain CP and radiation pattern diversities. To design a CP antenna, the major challenge is to achieve the equal amplitude and the requisite $90^{\circ}$ phase difference between the two-CLL radiating elements. In the Ant. $\alpha / \alpha^{\prime}$, due to the asymmetric structural configuration, two orthogonal degenerate modes are provided which makes the proposed antenna to excite in $\mathrm{CP}$ at distinct frequency. When the antenna is reconfigured from Ant. $\alpha$ to Ant. $\alpha^{\prime}$, the polarization alters from right hand to left hand $\mathrm{CP}$ at a specific frequency. When the antenna is reconfigured from these states, the radiation pattern is almost unchanged.

\section{Results and Discussions}

In this section, the proposed antennas with various design structures are manufactured, and the simulated and measured results of the input reflection coefficient and radiation characteristics are discussed and shown. The two prototypes of the antenna are shown in Fig. 3.

The switch reduction approach applied in the proposed prototype allows the possibility of performing measurements to fully characterize the properties of the antenna, presenting important information about maximizing reconfiguration ranges and about affiliation among frequency, polarization, and pattern reconfigurations rather than the work in [10], [12].

According to Tab. 1, the simulated and measured reflection coefficients $\left(\left|S_{11}\right|\right)$ for the frequency agility case are shown in Fig. 4(a), for the radiation pattern agility case are presented in Fig. 4(b), and for the polarization reconfigurability case are exhibited in Fig. 4(c). The behavior of the unloaded printed monopole (Ant. 0) is also shown in Fig. 4(a).

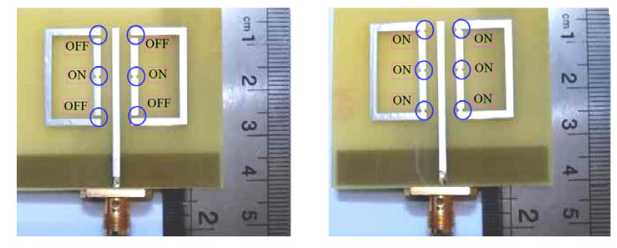

Fig. 3. Photographs of the fabricated proposed antennas.

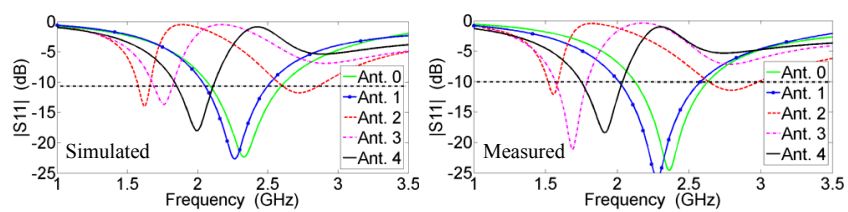

(a)

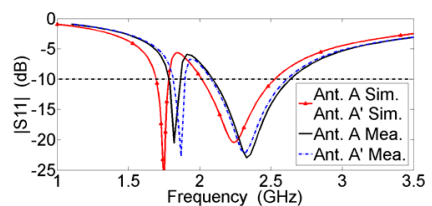

(b)

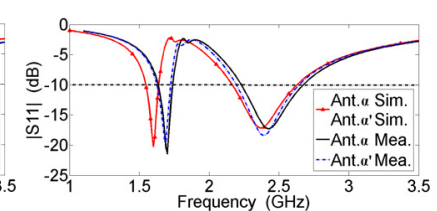

(c)
Fig. 4. Simulated and measured $\left|S_{11}\right|$ of the different switch configurations of the antenna, as listed in Tab. 1. (a) Frequency agility, (b) Pattern diversity, (c) Polarization reconfigurability.

A setup is used for frequency band reconfigurable structure to observe the measured $\left|\mathrm{S}_{11}\right|$ with the help of vector network analyzer and radiation characteristics by using anechoic chamber. The measured and simulated results are slightly different owing to material loss, fabrication tolerance and SMA connector. As shown in Fig. 4, the antenna operates at two resonance frequencies. The lower frequency corresponds to the resonance of the inductorloaded parasitic elements, whereas the second resonance corresponds to the antenna monopole. The operating frequency, $-10 \mathrm{~dB}$ impedance bandwidth and other parameters are listed in Tab. 2.

We can note from relation (1), when all of the switches are ON (Ant. 1), the CLL elements correspond to a closed loop and resonate at a much higher frequency, whereas its effective capacitance is almost zero. This resonance frequency occurs outside the band of interest. When the CLL elements are open by the gaps, they resonate at different frequencies for the various states. On the other hand, by modifying the effective inductances and capacitances of both the parasitic elements using the switches, the impedance matching of the entire antenna can be controlled. The resonance frequency ranges are lowered with a stepped increase of the capacitance and inductance values. For example, we have most effective capacitance and inductance for Ant. 2, so it has a lower resonance frequency $(1.55 \mathrm{GHz})$ rather than other states. For Ants. 1 up to 4 , the two CLLs operate at the same frequency because their structures are perfectly symmetric. Its frequency tuning range $T R=2\left(f_{\mathrm{oh}}-f_{\mathrm{ol}}\right) /\left(f_{\mathrm{oh}}+f_{\mathrm{ol}}\right) \times 100 \%=57.2 \%$, and its frequency tuning bandwidth $T B=2\left(f_{\max }-f_{\min }\right) /\left(f_{\max }+f_{\min }\right) \times$ $100 \%=65.8 \%$. In the above equation, $f_{\mathrm{ol}}$ and $f_{\mathrm{oh}}$ correspond, respectively, to the lowest and highest resonance frequencies; and $f_{\max }$ and $f_{\min }$ are, respectively, the maximum and 


\begin{tabular}{|c|c|c|c|c|c|c|c|c|c|c|}
\hline \multirow{2}{*}{$\begin{array}{l}\text { Mode/ } \\
\text { Antenna }\end{array}$} & \multicolumn{2}{|c|}{ Operation frequency } & \multicolumn{2}{|c|}{ Impedance bandwidth } & \multicolumn{2}{|c|}{$\begin{array}{c}\text { Simulated 3D radiation } \\
\text { patterns at }\end{array}$} & \multicolumn{2}{|c|}{$\begin{array}{l}\text { Measured Peak } \\
\text { Gain (dBi) at }\end{array}$} & \multicolumn{2}{|c|}{ Polarization at } \\
\hline & $\begin{array}{c}\mathrm{f}_{\mathrm{CLL}} \\
(\mathrm{GHz})\end{array}$ & $\begin{array}{c}f_{\text {mon }} \\
(\mathrm{GHz})\end{array}$ & $\begin{array}{l}\mathrm{BW}_{\mathrm{CLL}} \\
(\mathrm{GHz})\end{array}$ & $\begin{array}{l}\mathrm{BW}_{\text {mon }} \\
(\mathrm{GHz})\end{array}$ & $f_{C L L}$ & $\mathrm{f}_{\mathrm{mon}}$ & $\mathrm{f}_{\mathrm{CLL}}$ & $\mathrm{f}_{\mathrm{mon}}$ & $\mathrm{f}_{\mathrm{CLL}}$ & $f_{\text {mon }}$ \\
\hline Ant. 0 & - & $\begin{array}{c}2.33 \\
(2.34)^{*}\end{array}$ & - & $\begin{array}{c}2.07-2.62 \\
(2.1-2.6)\end{array}$ & & & - & 2.6 & - & LP \\
\hline Ant. 1 & - & $\begin{array}{c}2.26 \\
(2.22)\end{array}$ & - & $\begin{array}{c}2.04-2.52 \\
(1.97-2.54)\end{array}$ & & & - & 3.02 & - & LP \\
\hline Ant. 2 & $\begin{array}{c}1.62 \\
(1.55) \\
\end{array}$ & $\begin{array}{c}2.71 \\
(2.79) \\
\end{array}$ & $\begin{array}{c}1.56-1.67 \\
(1.51-1.59) \\
\end{array}$ & $\begin{array}{c}2.57-2.91 \\
(2.65-2.99) \\
\end{array}$ & & & 0.7 & 3.39 & LP & LP \\
\hline Ant. 3 & $(10,0)$ & $\begin{array}{l}1.76 \\
(1.7)\end{array}$ & $(10.010)$ & $\begin{array}{c}1.68-1.83 \\
(1.58-1.82)\end{array}$ & & & - & 3.24 & - & LP \\
\hline Ant. 4 & - & $\begin{array}{c}1.99 \\
(1.91) \\
\end{array}$ & - & $\begin{array}{c}1.83-2.12 \\
(1.75-2.05) \\
\end{array}$ & & & - & 3.12 & - & LP \\
\hline Ant. A & $\begin{array}{c}1.75 \\
(1.82)\end{array}$ & $\begin{array}{l}2.24 \\
(2.33)\end{array}$ & $\begin{array}{c}1.69-1.79 \\
(1.78-1.86)\end{array}$ & $\begin{array}{c}2.02-2.53 \\
(2.09-2.63)\end{array}$ & & & 1.20 & 3.46 & LP & LP \\
\hline Ant. $\mathrm{A}^{\prime}$ & $\begin{array}{c}1.75 \\
(1.87)\end{array}$ & $\begin{array}{l}2.24 \\
(2.32)\end{array}$ & $\begin{array}{l}1.69-1.79 \\
(1.82-1.9)\end{array}$ & $\begin{array}{l}2.02-2.53 \\
(2.08-2.62)\end{array}$ & & & 2.00 & 3.41 & LP & LP \\
\hline Ant. $\alpha$ & $\begin{array}{c}1.6 \\
(1.7)\end{array}$ & $\begin{array}{l}2.37 \\
(2.42)\end{array}$ & $\begin{array}{c}1.54-1.64 \\
(1.64-1.74)\end{array}$ & $\begin{array}{c}2.16-2.63 \\
(2.24-2.69)\end{array}$ & & & 0.95 & 3.55 & $\begin{array}{l}\mathrm{RH} \\
\mathrm{LP}\end{array}$ & LP \\
\hline Ant. $\alpha^{\prime}$ & $\begin{array}{c}1.6 \\
(1.69)\end{array}$ & $\begin{array}{l}2.37 \\
(2.38)\end{array}$ & $\begin{array}{c}1.54-1.64 \\
(1.63-1.72)\end{array}$ & $\begin{array}{l}2.16-2.63 \\
(2.2-2.65)\end{array}$ & & & 0.86 & 3.65 & $\begin{array}{l}\text { LH } \\
\text { LP }\end{array}$ & LP \\
\hline
\end{tabular}

Tab. 2. The results for the different switch configurations of the antenna. (*The values within braces indicate measured results).

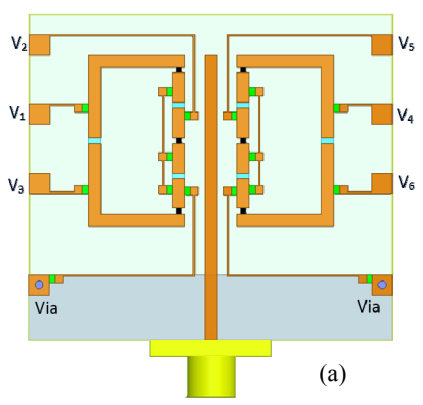

Fig. 5. (a) The antenna structure by considering the actual effect of biasing circuit and pin diode, (b) Equivalent $\mathrm{RF}$ circuit of the PIN diode in ON and OFF states.

minimum frequencies satisfying $\left|\mathrm{S}_{11}\right|<-10 \mathrm{~dB}$. These definitions are taken from [20]. The operating frequency and impedance bandwidth of the monopole influenced using the mismatch made by the presence of the two pairs of parasitic elements at the right and left sides of it, compared to Ant. 0. Ants. 1 up to 4 act essentially as an LP radiator with almost symmetrical and like a monopole radiation pattern of their operating frequencies.

In the folowing, the effect of biasing circuit and a real model of a pin diode on the $\left|\mathrm{S}_{11}\right|$ of the proposed antenna compared with the ideal switch is separately investigated. Figure 5(a) shows the antenna structure with a bias circuit network. For this purpose, six low-cost PIN diode (BAR $50-02 \mathrm{v})$ as switch, sixteen surface-mount inductors $(56 \mathrm{nH})$ as RF chokes, and six surface-mount capacitors $(100 \mathrm{pF})$ as $\mathrm{DC}$ isolation are used in the structure. To control the status of the diodes, six independent DC bias voltages $\left(V_{1}, V_{2}, V_{3}\right.$, $V_{4}, V_{5}$ and $V_{6}$ ) in six terminals are used. In the bias circuit, two vias on either side of the structure are used to connect to the ground.

The non-ideal diodes as "switches" have an additional loading effect on the antenna performance, which needs to be taken into account. This effect can be modelled by the $\mathrm{RF}$ equivalent circuit of the diodes provided by the manufacturing company. The circuit model used in the full-wave simulations in the ON/OFF states is shown in Fig. 5(b). As can be seen, both the ON and OFF states have the packaging inductive effect of inductance $L$. The equivalent circuit in the ON state (forward biased) has a low forward resistance $R_{\mathrm{S}}$ which contributes to the insertion loss and is the most limiting factor for this state.

The equivalent circuit for OFF state (zero or reverse biased) has the parallel combination of the parallel reversed bias resistance $R_{\mathrm{P}}$ and the total capacitance $C_{\mathrm{T}}$, which contributes to the isolation. All of the values necessary to form the circuit models are given in a standard PIN diode datasheet. Based on the PIN diode datasheet [21], it has a parallel circuit with a resistance of about $R_{\mathrm{P}}=5 \mathrm{k} \Omega$ and a capacitance of $C_{\mathrm{T}}=0.15 \mathrm{pF}$ for the OFF state and a forward resistance of $R_{\mathrm{S}}=3 \Omega$ for the ON state. The series parasitic inductance due to chip packaging is only $L_{1}=0.6 \mathrm{nH}$ for this diode and hence it can be neglected for simplicity.

Figure 6 shows a comparison between $\left|S_{11}\right|$ simulation results in the real and ideal model of the pin diodes for Ant. A and Ant. $\alpha$. As it is clear from the figure, the real pin diodes affect on the electrical length of the antenna somehow the desired operating frequency may have suffered displacement and they have been effective at the level of return loss.

The measured radiation patterns are represented in Fig. 7 for the Ant. 1 and Ant. 2 over the YZ- and XZplanes with vertical polarization (same as the driven monopole). The Ant. 3 and Ant. 4 have similar radiation characteristics. The measured patterns are effectively steered towards the boresight directions and the peak gain is between (3.02-3.39) dB for all frequencies, as listed in Tab. 2. 


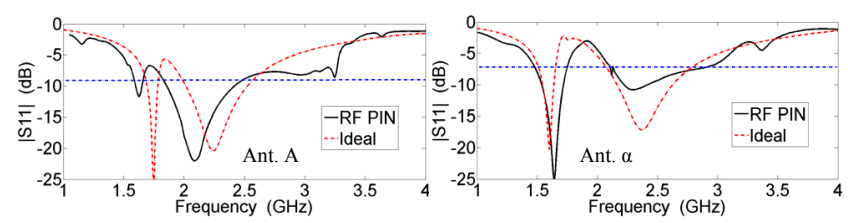

Fig. 6. The PIN diode effects on the behavior of resonant antenna compared with the ideal switch.

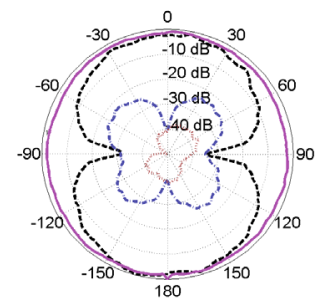

Ant. 1 at $2.34 \mathrm{GHz}$

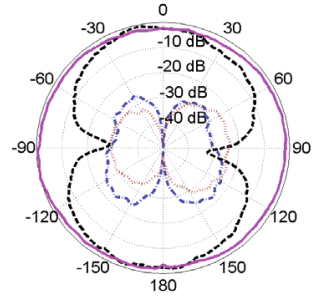

Ant. 2 at $1.55 \mathrm{GHz}$

$$
\begin{array}{|l|}
\hline--X Z \text {-plane, Co pol. } \\
-\cdots \text { YZ-plane, Cross pol. } \\
\text { - YZ-plane, Co pol. } \\
\text { XZ-plane, Cross pol. }
\end{array}
$$

Fig. 7. Measured radiation patterns of the Ant. 1 and Ant. 2 in YZ- and XZ-planes at their operating frequencies.

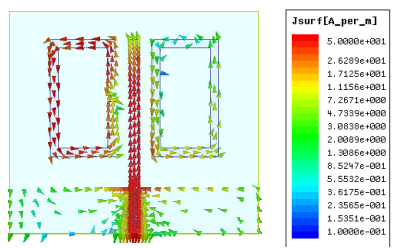

(a)

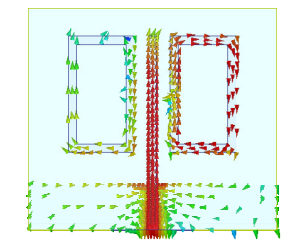

(b)
Fig. 8. Simulated surface current at the $f_{\mathrm{L}}=1.75 \mathrm{GHz}$ for the (a) Ant. A, (b) Ant. A'.

It is shown from the figures that radiation characteristics are not almost altered while the antenna operation is in the frequency diversity mode. Therefore, the antenna is able to tune its operation frequency/band and maintains the radiation pattern totality. It is worth mentioning that the actual model of the pin diode switch rather than ideal switch has little effect on the performance of antenna radiation. Therefore, the comparison of them is ignored.

For Ants. A/A', when the switch status is such that one CLL is a closed loop and the other one has the gaps, the lower resonance frequency related to the single-CLLloaded monopole, whereas the monopole operation is affected a little. Therefore, it can be made a radiation pattern pointing either along the negative or positive y-axis, depending on the active CLL. It is shown in Tab. 2 that the patterns related to the two excited CLLs point toward various directions. These pattern changes are correlated to the sweeping changes in the current distributions along the vertical strips (the monopole and the sides of the CLL elements parallel to the $\mathrm{x}$-axis). In order to give a physical insight into the behavior of the antenna for pattern diversity, the current distributions of the Ants. A and $\mathrm{A}^{\prime}$ shown in Fig. 8(a) and 8(b) at the same resonance frequency $f_{\mathrm{L}}=1.75 \mathrm{GHz}$, respectively. We notice that the current distributions, as would be expected, are symmetric. This behavior confirms the previous discussion of the coincident

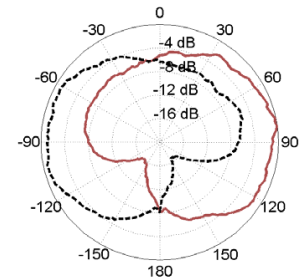

$\mathrm{xy}$-plane at $1.84 \mathrm{GHz}$

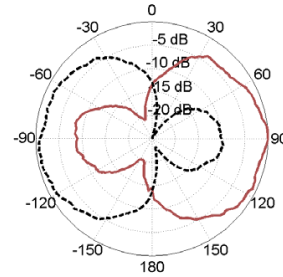

yz-plane at $1.84 \mathrm{GHz}$

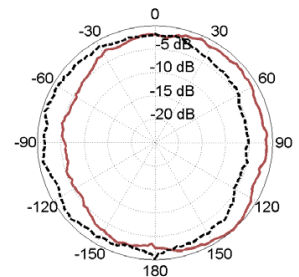

xy-plane at $2.33 \mathrm{GHz}$

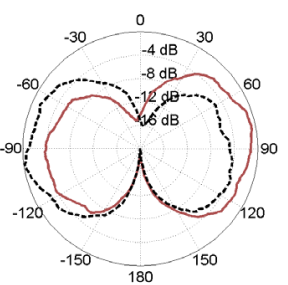

yz-plane at $2.33 \mathrm{GHz}$
Fig. 9. Measured radiation pattern in xy- and yz-planes for the Ant. A (Solid lines) and Ant. A' (dashed lines).
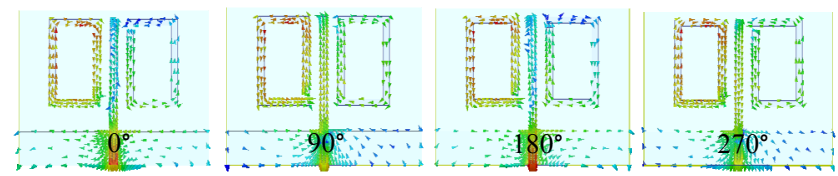

Fig. 10. Surface current distribution of the antenna in the Ant. $\alpha$ during one time cycle T.

$\left|\mathrm{S}_{11}\right|$ results at the same frequency.

The measured radiation patterns in xy-plane (azimuth cut) and yz-plane (elevation cut) for Ant. A/A' are shown in Fig. 9. The radiation patterns in the xz-plane are almost unchanged for these states, so they are not shown for brevity. It can be seen that the cross polarization level is very minor. It is also observed that frequency characteristics are maintained in these states, while changing the beam direction when the CLL elements are switched, which satisfies the principle of pattern reconfigurability and depicting good diversity performance.

However, when the antenna is switched to Ants. $\alpha / \alpha^{\prime}$, asymmetry is presented in the basic configuration altering the current flow direction on the CLL surfaces. Especially, owing to the presence of left CLL, the basic of current is agitated by altering the vector of the surface current in $\mathrm{y}$ direction without changing vector of current directed along $\mathrm{x}$-direction. This makes the resonant mode of current bisection into two orthogonal degenerated modes with $90^{\circ}$ phase shift and equal amplitude. Therefore, the instantaneous vector of the surface current on the CLL rotates and the radiated fields are circularly polarized. The vector direction of current is anticlockwise and thus the antenna has RHCP pattern for Ant. $\alpha$. Similarly, in Ant. $\alpha^{\prime}$, the right CLL alters the perturbation of current and the two degenerated orthogonal modes have $-90^{\circ}$ phase shift and equal amplitude which makes the vector of the surface current rotate in clockwise direction generating LHCP radiation pattern. The distribution curves of surface current for Ant. $\alpha$ operation in phase of $0^{\circ}, 90^{\circ}, 180^{\circ}$, and $270^{\circ}$ are shown in Fig. 10. In this reconfiguration process, as shown in Tab. 2 , 


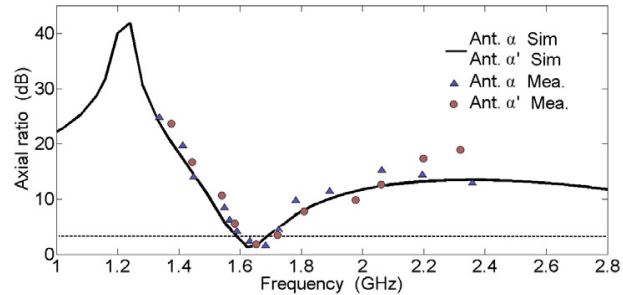

Fig. 11. Simulated and measured axial ratios of Ant. $\alpha / \alpha^{\prime}$.

\begin{tabular}{|c|c|c|}
\hline Mode/Antenna & Simulated & Measured \\
\hline Ant. $\alpha$ & $1.57-1.67 \mathrm{GHz}$ & $1.59-1.72 \mathrm{GHz}$ \\
\hline Ant. $\alpha^{\prime}$ & $1.57-1.64 \mathrm{GHz}$ & $1.6-1.72 \mathrm{GHz}$ \\
\hline
\end{tabular}

Tab. 3. Antenna CP bandwidth results.

the beam direction of radiation patterns and resonance frequency are also unchanged.

The simulated and measured axial ratios of Ant. $\alpha / \alpha^{\prime}$ are shown in Fig. 11. Satisfactory agreements are obtained between the measured and simulated results. The discrepancy compared with the simulation may come from several aspects such as the influence of the fabrication error. Due to structure symmetry, the RHCP and LHCP modes have the similar results at the same working frequency. This can be observed from Fig. 11, where axial ratio in Ant. $\alpha$ is almost the same as in Ant. $\alpha^{\prime}$. The obtained CP bandwidths, referred to $3-\mathrm{dB} \mathrm{AR}$, assessed from simulation results in comparison to measurement results are tabulated in Tab. 3 . It has to be important that the impedance bandwidths in Tab. 2 totally cover the corresponding AR bandwidth in Tab. 3. Consequently, the available working bandwidth is mainly determined by the AR bandwidth as the proposed reconfigurable antenna performs polarization switching at one frequency.

Figure 12 shows the measured radiation patterns in the xz- and yz-planes for Ants. $\alpha / \alpha^{\prime}$. It is also seen that the pattern is tilted off-broadside by about $30^{\circ}$. This beam tilt is due to the asymmetrical configuration of the two CLL-
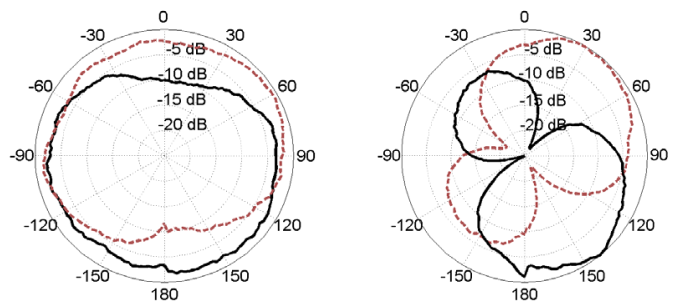

Ant. a, yz-plane at $1.65 \mathrm{GHz} \quad$ Ant. $a, x z$-plane at $1.65 \mathrm{GHz}$

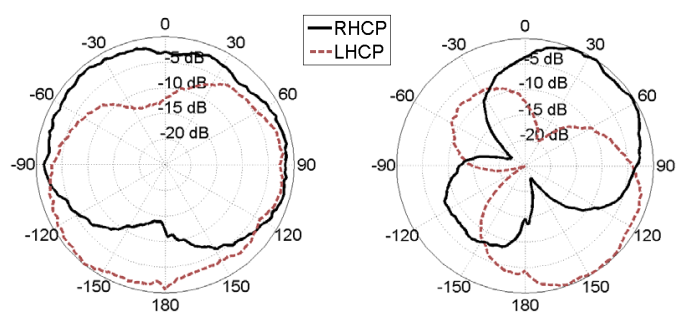

Ant. $\alpha^{\prime}, y z-$ plane at $1.65 \mathrm{GHz}$ Ant. $\alpha^{\prime}, x z-$ plane at $1.65 \mathrm{GHz}$

Fig. 12. Measured radiation pattern in the xz- and yz-planes for Ants. $\alpha / \alpha^{\prime}$.

\begin{tabular}{|c|c|c|c|c|c|}
\hline Ref. & $\begin{array}{c}\text { Substrate } \\
\text { \& size } \\
\left.\mathbf{( m m}^{\mathbf{2}}\right)\end{array}$ & $\begin{array}{c}\text { Switching } \\
\text { element }\end{array}$ & $\begin{array}{c}\text { Operation } \\
\text { frequency } \\
\text { (GHz) }\end{array}$ & $\begin{array}{c}\text { Operation } \\
\text { radiation } \\
\text { beams }\end{array}$ & $\begin{array}{c}\text { Operation } \\
\text { polarization }\end{array}$ \\
\hline$[10]$ & $\begin{array}{c}\text { RO-TMM } \\
\& 50 \times 50\end{array}$ & $\begin{array}{c}\text { Over } 24 \\
\text { MEMS }\end{array}$ & $4-7$ & $0-90^{\circ}$ y-dir & LP \\
\hline$[14]$ & $\begin{array}{c}\text { Ro } 5870 \\
\& 66 \times 66\end{array}$ & $\begin{array}{c}24 \text { Pin } \\
\text { diodes }\end{array}$ & $2.25-2.4$ & \pm z-dir. & LP,RH/LHCP \\
\hline$[22]$ & $\begin{array}{c}\text { RO4350 \& } \\
65 \times 65\end{array}$ & $\begin{array}{c}6 \text { Pin } \\
\text { diodes }\end{array}$ & $1.6-2$ & Not state & LP, RH/LHCP \\
\hline $\begin{array}{c}\text { This } \\
\text { work }\end{array}$ & $\begin{array}{c}\text { FR4 \& } \\
45 \times 40\end{array}$ & 6 switch & $1.5-2.9$ & $\pm 30^{\circ}$ y-dir. & LP, RH/LHCP \\
\hline
\end{tabular}

Tab. 4. Comparison between proposed and some reported reconfigurable antennas.

loaded elements, compared to two CLL elements which have a symmetrical configuration and shows a beam of broadside. The major polarization $\mathrm{Z}>0$ is the LHCP and the minor is the RHCP for $Z<0$ in Ant. $\alpha$, and it is inverse for Ant. $\alpha^{\prime}$.

Table 4 shows the comparison between the proposed antenna characteristics with some reported reconfigurable antennas. It indicates that this work has an advantage of wider bandwidth across radiation pattern reconfiguration with low profile size.

\section{Conclusion}

Simple, efficient, multi-reconfigurable metamaterialinspired antenna was obtained by integrating six switches into its two CLL-based NFRP elements. Eight structures were examined by considering the gap states in the CLL elements, whether they were closed or open. The resulting performance characteristics such as operating frequency, impedance bandwidth, polarization, and radiation pattern were reported. In fact, good agreement between the measured and simulated results was presented. The proposed structure led to a design extension that was demonstrated to have a much wider frequency-agile bandwidth, i.e., $65.8 \%$. Simulation and measurement results show that the structure can also alter its beam direction by changing the switched states in the \pm y-direction. Two polarization selective states are also designed with polarization reconfigurations from RHCP to LHCP. The designed antenna, thus, represents a good candidate for wireless application providing smart countermeasures in highly interfered channels and multipath affected.

\section{References}

[1] OJAROUDI, N., BASHERLOU, H. J., et al. Recent developments of reconfigurable antennas for current and future wireless communication systems. Electronics, 2019, vol. 8, p. 1-17. DOI: 10.3390/electronics 8020128

[2] FAKHARIAN, M. M., REZAEI, P., OROUJI, A. A. A novel slot antenna with reconfigurable meander-slot DGS for cognitive radio applications. Applied Computational Electromagnetics Society (ACES) Journal, 2015, vol. 30, no. 7, p. 748-753. 
[3] GE, L., LI, M., LI, Y., et al. Linearly polarized and circularly polarized wideband dipole antennas with reconfigurable beam direction. IEEE Transactions on Antennas and Propagation, 2018, vol. 66 , no. 4, p. 1747-1755. DOI: 10.1109/TAP.2018.2797520

[4] QIN, P.-Y., GUO, Y., WEILY, A. R., et al. A pattern reconfigurable U-slot antenna and its applications in MIMO systems. IEEE Transactions on Antennas and Propagation, 2012, vol. 60 , no. 2, p. 516-528. DOI: 10.1109/TAP.2011.2173439

[5] FAKHARIAN, M. M., REZAEI, P., OROUJI, A. A., et al. A wideband and reconfigurable filtering slot antenna. IEEE Antennas and Wireless Propagation Letters, 2016, vol. 15, p. 1610-1613. DOI: 10.1109/LAWP.2016.2518859

[6] FAN, Y., LI, R. L., CUI, Y. Development of polarisation reconfigurable omnidirectional antennas using crossed dipoles. IET Microwaves, Antennas and Propagation, 2019. vol. 13, no. 4, p. 485-491. DOI: 10.1049/iet-map.2018.5490

[7] FAKHARIAN, M. M., REZAEI, P., OROUJI, A. A. Polarization and radiation pattern reconfigurability of a planar monopole-fed loop antenna for GPS application. Radioengineering, 2016, vol. 25, no. 4, p. 680-686. DOI: 10.13164/re.2016.0680

[8] TAWK, Y., EL-AMINE, A., SAAB. S., et al. A software-defined frequency-reconfigurable meandered printed monopole. IEEE Antennas and Wireless Propagation Letters, 2018, vol. 17, no. 2, p. 327-330. DOI: 10.1109/LAWP.2017.2788461

[9] SBOUI, F., MACHAC, J., GHARSALLAH, A. Low-profile slotted SIW cavity backed antenna for frequency agility. Radioengineering, 2019, vol. 28, no. 2, p. 386-390. DOI: $10.13164 /$ re.2019.0386

[10] GRAU BESOLI, A., DE FLAVIIS, F. Multifunctional reconfigurable pixeled antenna using MEMS technology on printed circuit board. IEEE Transactions on Antennas and Propagation, 2011, vol. 59, no. 12, p. 4413-4424. DOI: 10.1109/TAP.2011.2165470

[11] FAKHARIAN, M. M., REZAEI, P., OROUJ, A. A. Reconfigurable multiband extended U-slot antenna with switchable polarization for wireless applications. IEEE Antennas and Propagation Magazine, 2015, vol. 57, no. 2, p. 194-202. DOI: 10.1109/MAP.2015.2414665

[12] RODRIGO, D., CETINER, B. A., JOFRE, L. Frequency, radiation pattern and polarization reconfigurable antenna using a parasitic pixel layer. IEEE Transaction on Antennas and Propagation, 2014, vol. 62, no. 6, p. 3422-3427. DOI: 10.1109/TAP.2014.2314464

[13] SULAKSHANA, C., ANJANEYULU, L. A compact reconfigurable antenna with frequency, polarization and pattern diversity. Journal of Electromagnetic Waves and Applications, 2015, vol. 29, no. 15, p. 1953-1964. DOI: 10.1080/09205071.2015.1068229

[14] GE, L., LI, Y., WANG, J., et al. A low-profile reconfigurable cavity-backed slot antenna with frequency, polarization, and radiation pattern agility. IEEE Transactions on Antennas and Propagation, 2017, vol. 65, no. 5, p. 2182-2189. DOI: 10.1109/TAP.2017.2681432

[15] ZIOLKOWSKI, R. W., JIN, P., LIN, C.-C. Metamaterial-inspired engineering of antennas. Proceedings of the IEEE, 2011, vol. 99, no. 10 , p. $1720-1731$. DOI: 10.1109/JPROC.2010.2091610

[16] DONG, Y., ITOH, T. Metamaterial-based antennas. Proceedings of the IEEE, 2012, vol. 100, no. 7, p. 2271-2285. DOI: 10.1109/JPROC.2012.2187631

[17] JIN, P., ZIOLKOWSKI, R. W. Multi-frequency, linear and circular polarized, metamaterial-inspired, near-field resonant parasitic antennas. IEEE Transactions on Antennas and Propagation, 2011, vol. 59, no. 5, p. 1446-1459. DOI: 10.1109/TAP.2011.2123053
[18] DAKHLI, S., RMILI, H., FLOC'H, J.-M., et al. Capacitively loaded loop-based antennas with reconfigurable radiation patterns. International Journal of Antennas and Propagation, 2015, p. 1-10. DOI: $10.1155 / 2015 / 523198$

[19] BARBUTO, M., BILOTTI, F., TOSCANO, A. Design of a multifunctional SRR-loaded printed monopole antenna. International Journal of $R F$ and Microwave Computer-Aided Engineering, 2012, vol. 22, no. 4, p. 552-557. DOI: 10.1002/mmce. 20645

[20] TANG, M.-C., ZIOLKOWSKI, R. W. Frequency-agile, efficient, circularly polarized, near-field resonant antenna: designs and measurements. IEEE Transactions on Antennas and Propagation, 2015, vol. 63, no. 11, p. 5203-5209. DOI: 10.1109/TAP.2015.2477563

[21] INFINEON TECHNOLOGIES, BAR50 Silicon PIN Diode, (datasheet). 16 pages. [Online] Cited 2011-07-18. Available at: http://www.alldatasheet.com/datasheetpdf/pdf/78976/ININEON/B AR5002.html.

[22] PRIYA, A., KAJA MOHIDEEN, S., SARAVANAN, M. Multistate reconfigurable antenna for wireless communications. Journal of Electrical Engineering and Technology, 2020, vol. 15, p. 251-258. DOI: $10.1007 / \mathrm{s} 42835-019-00321-8$

\section{About the Authors ...}

Mohammad M. FAKHARIAN was born in Tehran, Iran, in 1987. He received the B.S., M.S. and Ph.D. degrees in Electrical Engineering from Semnan University, Semnan, Iran, in 2009, 2012 and 2016, respectively. Currently, he is assistant professor in the University of Garmsar, Garmsar, Iran. His research interests include low-profile printed and patch antennas for wireless communication, fractal, miniature and multiband antennas, meta-materials and EBG structures interaction with antennas and RF passive components, reconfigurable antennas, and electromagnetic theory: numerical methods and optimization techniques.

Pejman REZAEI was born in Tehran, Iran, in 1977. He received the B.S. degree in Electrical-Communication Engineering from Communication Faculty, Tehran, Iran, in 2000, and the M.S. and Ph.D. degrees from Tarbiat Modarres University, Tehran, Iran, in 2002 and 2007, respectively. Currently, he is associated professor in the Semnan University, Semnan, Iran. His current research interests are electromagnetics theory, theory and design of antenna, reconfigurable antenna, metamaterial structure, and satellite communication.

Ali A. OROUJI was born in Neyshabour, Iran, in 1966. He received the B.S. and M.S. degrees in Electronic Engineering from Iran University of Science and Technology, Tehran, Iran, in 1989 and 1992, respectively, and the Ph.D. degree from the Indian Institute of Technology Delhi, Delhi, India, in 2006. Since 1992, he has been a Faculty Member with Semnan University, Semnan, Iran. His research interests are in modeling of silicon-on-insulator metal-oxide-semiconductor field-effect transistors, novel device structures, theory and design of antenna, and wave propagation. 